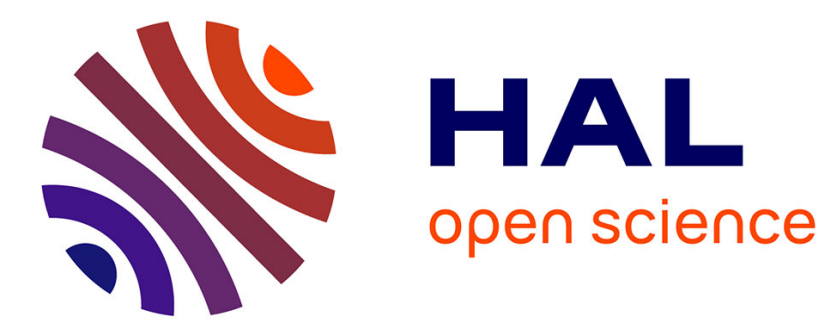

\title{
Meta-Synthetic Methodology: A New Way to Study Agricultural Rumor Intervention
}

Ruya Tian, Lei Wu, Yijun Liu, Xuefu Zhang

\section{To cite this version:}

Ruya Tian, Lei Wu, Yijun Liu, Xuefu Zhang. Meta-Synthetic Methodology: A New Way to Study Agricultural Rumor Intervention. 9th International Conference on Computer and Computing Technologies in Agriculture (CCTA), Sep 2015, Beijing, China. pp.375-389, 10.1007/978-3-319-483573_37. hal-01557829

\author{
HAL Id: hal-01557829 \\ https://hal.inria.fr/hal-01557829
}

Submitted on 6 Jul 2017

HAL is a multi-disciplinary open access archive for the deposit and dissemination of scientific research documents, whether they are published or not. The documents may come from teaching and research institutions in France or abroad, or from public or private research centers.
L'archive ouverte pluridisciplinaire HAL, est destinée au dépôt et à la diffusion de documents scientifiques de niveau recherche, publiés ou non, émanant des établissements d'enseignement et de recherche français ou étrangers, des laboratoires publics ou privés.

\section{(c)(1)}

Distributed under a Creative Commons Attribution| 4.0 International License 


\title{
Meta-Synthetic Methodology: A New Way to Study Agricultural Rumor Intervention
}

\author{
Ruya Tian ${ }^{1,2, \mathrm{a}}$, Lei Wu ${ }^{1,2, \mathrm{~b}}$, Yijun Liu ${ }^{3, \mathrm{c}}$, Xuefu Zhang ${ }^{1,2, \mathrm{~d}, *}$ \\ ${ }^{1}$ Agricultural Information Institute, Chinese Academy of Agricultural Sciences (CAAS), \\ Beijing, 100081, P. R. China; \\ ${ }^{2}$ Key Laboratory of Agri-information Service Technology, Ministry of Agriculture, Beijing \\ 100081, P. R. China; \\ ${ }^{3}$ Institute of Policy and Management, Chinese Academy of Sciences, Beijing, 100190, P. R. \\ China \\ ªtianruya@126.com, birlrable@126.com, ${ }^{\mathrm{c} y i j u n l i u @ c a s i p m . a c . c n, ~}{ }^{\mathrm{d}}$ zhangxuefu@caas.cn
}

\begin{abstract}
Intervention of online public opinion, especially agricultural rumors, has increasingly become an important issue that is related to the online society safety and reflecting the managers' social management capabilities. In this paper, meta-synthetic methodology was used to investigate the intervention mechanism of online public opinion system. General characteristics of online public opinion system were characterized qualitatively, and an opinion supernetwork model was built to analyze the intervention mechanism quantitatively. We put forward a rumor intervention mechanism including intervention timing, intervention mode, and intervention intensity. Based on system modeling and simulation, an agricultural rumor case was used to verify the practical effects of online agricultural rumor intervention. The results show that rumor intervention based on meta-synthetic methodology produces good results. This investigation will help to provide a reliable basis for the development of more sophisticated and effective rumor intervention strategies.
\end{abstract}

Keywords: meta-synthetic methodology, online public opinion system, agricultural rumor intervention, intervention mechanism

Corresponding Author: Xue-Fu Zhang, +8610-82106252, zhangxuefu@caas.cn 


\section{Introduction}

With the popularity of the web, BBS and Twitter on Internet have increasingly become the most important channel for people to publish, exchange and access to information. It is now an important platform for Internet users to leave comments on emergencies, to express attitudes and to vent their emotions. Furthermore, it even becomes an important force in promoting the development of the incidents. Monitoring and management of agricultural rumors are increasingly becoming an important aspect of the national interests and a matter of national security, which make the emergency management of the online rumors become a hot spot [1-4]. At present, researches on online public opinion intervention in China focused more on the qualitative ways, which takes case analysis as the main method. While scientific, systematic, and quantitative research is rare [5].

System is the general form of the existence of things. One of the founders of general systems theory, Bertalanffy, defined the system as "A synthesis of all related factors", which is an organic integrity with a specific function combined of several interactional and interdependent components [6]. General systems theory requires people to see things from a global standpoint, to comprehensively analyze the relationships between the elements in the system, elements and their system, system and its environment, this system and other systems. By doing so, internal relations and laws of a system can be grasped, as well as the way to control and transform it effectively. Meta-synthetic methodology proposed by the Chinese famous scholar Xue-Sen Qian, illustrates a comprehensive approach of qualitative and quantitative methods [7]. It is a creative sublimation to systems theory, providing a new way of thinking and methods to solve the problem of open complex giant system [8].

This paper investigated the intervention mechanism of online public opinion from a meta-synthetic methodological point of view. Online public opinion can be seen as a system. It has general constituent elements. Each element in the system is on a particular position with specific functions. Links and constraints between elements of each other constitute an indivisible whole. On the basis of elaborating its composition, structure, and function qualitatively, this paper quantified the intervention mechanism for online public opinion to provide a new research perspective for online public opinion intervention.

The rest of this paper is organized as follows. Section 2 describes online public opinion system, including its composition, structure, and function. In Section 3, we 
built an Opinion SuperNetwork according to the system characteristics to explore the online public opinion, and then discussed the intervention mechanism based on this supernetwork. In Section 4, we applied the methods described in Section 3 to a certain food safety event, and the results are presented and compared. Section 5 concludes, and suggests possible future research approaches.

\section{Online Public Opinion System}

Online public opinion is a system of particular composition, structure, and function. Intervention and guide for online public opinion can be highly effective or even gets twice the result with half the effort by fully considering the inherent properties and operation mechanism of the online public opinion system. Online public opinion system is the sum of beliefs, attitudes, opinions and emotions, etc. It is public opinion subject published on the Internet about certain social events under certain psychologies at a certain time and space. It can impact on the development of the events.

Online public opinion system consists of opinion subjects, opinion objects, and system dynamics, as is shown in Fig. 1. Public opinion system exists in a particular system environment, and is divided from its environment by the system boundary. There are certain inputs and outputs between the public opinion system and its environment. Opinion subjects of public opinion system includes the opinion agents discussing the events and the parties of the events. For instance, Netizens are opinion subjects in public opinion system. The opinion object is the sum of beliefs, attitudes, opinions and emotions, etc., which are published by public opinion subjects on the Internet, such as netizens' posts. System dynamic is the psychology of public opinion subjects, which is the internal driving force for subjects to express different objects. For example, hatred answers for the deep psychological reason of certain netizens who publish rumors. 


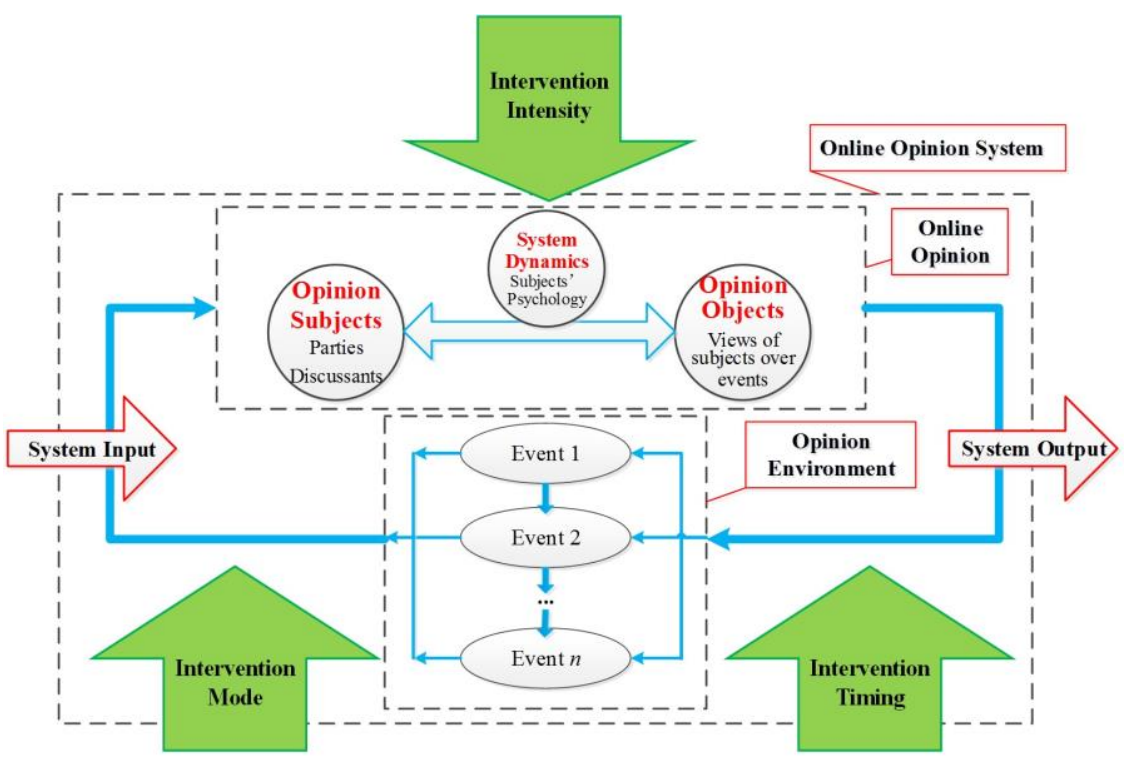

Fig. 1. Online public opinion system and its intervention mechanism

Public opinion system environment is the event evolvement, which is the input of the system, and also the external driving force of the development of the system. System boundary is the certain space and time, as well as specific event. Effect of opinion system on the event is the system output. There are two fundamentally different types of system output. One is the direct result caused by the system. The other type is the conclusion with more universal significances. As in "Guo Mei-mei rumor event", the direct result of the online public opinion system is Guo Mei-mei clarified that there is no relationship between her and the Chinese Red Cross, and Red Cross disclosed its money flows. While universal conclusions are we need to reflect on the values of the post-90s generation, to explore methods resolving the government confidence crisis, and to ponder the role of the media.

Relation mode between various elements of the opinion system is the system's structure. First, opinion subjects interact one another. For instance, opinion of the opinion leaders influences the others' opinion [9-10]. Second, there are temporal and spatial dependencies between opinion objects. Some posts published earlier on time result in preconception impacts on later posts. And information nonidentity of different forums caused the isolation between the opinion objects in space. Thirdly, there is a relationship of action and reaction between opinion subject and object. Subjects express different viewpoints under different system dynamics, and different 
viewpoints in turn affect the psychology of the subjects. Last but not least, system dynamic is the connector, mediator, and promoter associating system subjects and objects. It is the internal driving force of system development and evolvement. Interactions between this subject and that subject, this object and that object, as well as between subject and object are driven by the system dynamic.

Online public opinion impacts on its environment, i.e. the evolvement of incidents. This is the output of the public opinion system. It can reflect the function of the system. Discussions in online public opinion system have positive effects on the development of the event. It is a collection and a reflection of public opinion. Online public opinion system is a promoter for government policy to be transparent and open. But the negative impacts of the online public opinion should not be overlooked.

First, the online public opinion can sometimes become an amplifier of false information and rumors, leading to misinformation and opinion anomie. Internet is a place mixed of good and evil. People with different ages, professions, and interests gathered in this discourse space. Anyone can feel free to post or retweet the information of social events after he or she registered an ID as a forum user or had a personal twitter, which opens the door for false information and rumors.

Second, the online public opinion can be the fuse of public discontent sometimes, with negligible impact on social stability. In the online public discourse space, multi-dimensional interacts among Internet users include not only text, but also an emotional interaction. It is noteworthy that, discontent of netizens often start from criticizing a social event, and be further aggravated with the criticism going deeper. It sometimes bring bad impacts on social stability.

Third, the online public opinion can sometimes become a splitter digesting social cohesion, which leads to social crisis of confidence. With the increase of online rumor, social confidence is facing a deeper crisis. In recent years, the social crisis of confidence problems caused by the spread of online rumor is worth more increasingly of concern.

\section{Intervention Mechanism of Online Public Opinion System}

Qualitative descriptions for components, structure, and function of the online public opinion system provide a theoretical basis for quantitative calculation of system mechanism. Online public opinion intervention can be implemented based on the characteristics of the system. Regulating the composition and structure of the system 
changes function of the system. This will make public opinion interacting with the event positively and achieve the goal of rumor intervention.

\subsection{Online Public Opinion System Modeling}

A supernetwork is defined as a network that exists above and beyond existing networks [11]. In recent years, it is applied in many aspects such as knowledge networks [12], logistics (supply chain) [13], traffic [14], ecology [15], and so on. According to the compositional and structural characteristics of online public opinion system, a supernetwork model including Social Subnetwork, Environment Subnetwork, Psychological Subnetwork, and Viewpoint Subnetwork can be created (Fig. 2). The model in this study can not only depict the relationships between the elements in the public opinion formation process, but better reveal the dynamics and evolution mechanisms of the system [16-17].

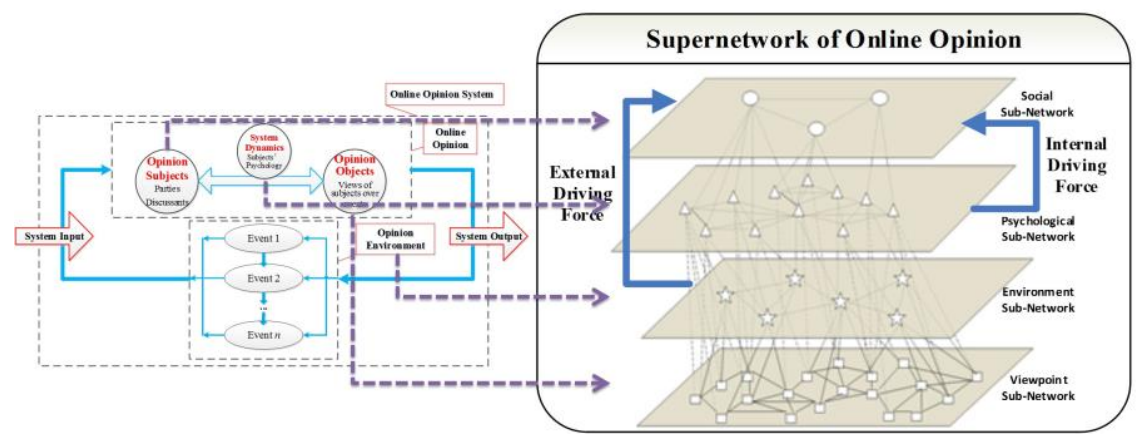

Fig. 2. Online public opinion system modeling

\subsubsection{Social Subnetwork}

Take the nodes of the network for the agents issued public opinion, and the network edges for the reply relationships between the agents. By doing so, we form the "Social Subnetwork of Opinion SuperNetwork $\left(G_{A}\right)$ ".Opinion agents who issued positive viewpoints are positive agents in the public opinion system. The one who issued negative viewpoints are negative agents.

\subsubsection{Environment Subnetwork}


Take the nodes of the network for the environments under which public opinion issued, and the network edges for relationships between issued time of pieces of information. By doing so, we form the "Environment Subnetwork of Opinion SuperNetwork $\left(G_{E}\right)^{\prime}$. A negative environment is the one with rumor spreading, while positive environment refutes rumor spreading.

\subsubsection{Psychological Subnetwork}

Take the nodes of the network for agents' psychology who issued public opinion, and the network edges for their transformation relationships. By doing so, we create the "Psychological Subnetwork of Opinion SuperNetwork $\left(G_{P}\right)$ ". The agents with positive psychology are reluctant to believe the rumors, and the ones with negative psychology are inclined to believe rumors.

\subsubsection{Viewpoint Subnetwork}

Take the nodes of the network for viewpoint keywords issued by public opinion agents, and network edges for various keywords' affiliation relationships. By doing so, we create the "Viewpoint Subnetwork of Opinion SuperNetwork $\left(G_{K}\right)$ ". The viewpoint with rumor spreading is negative, while the one with rumor clarifying is positive.

\subsubsection{Opinion SuperNetwork}

Take opinion agents, opinion environment, agent's psychology and viewpoints as nodes, the relationships in and between the Subnetworks for superedges ( $S E$ ) to create "Opinion SuperNetwork (OSN)".

$$
O S N=\left(G_{A}, G_{E}, G_{P}, G_{K}, S E_{a-e-p-k}\right)
$$

This model can be used to describe the overall features and the general rules of online public opinion system. Based on this model, we explore the quantitative intervention mechanisms, including how to make the best timing to intervene, select feasible intervention mode, as well as master the appropriate intervention intensity (Fig. 1). 


\subsection{Intervention Timing}

Currently, the intervention of online agricultural rumor is implemented after the event had become opinion hotspot. Government departments are involved in the interference with netizens' urgent requirement, missed the opportunity to change the negative momentum of the public opinion. This often caused netizens' emotion siltation and conditions that public opinion is detrimental to the development of the event. Due to time rush, a lot of intervention agents often have too little expertise to manage the crisis. Researchers have proved that it is better to manage public opinion crisis with responding to the crisis within "golden four hours" [18].

It is an ideal choice to implement intervention according to the characteristics of the online public opinion system, before system output, i.e. before the system caused impact or significant influence to the event. So quantitative prediction of system output is particularly important. Superlink (i.e. superedges) prediction referred to the prediction of existent yet unknown superlinks and future superlinks in a supernetwork structure [19]. Superlink prediction between opinion agents can be conducted by method shown in Fig. 3 [20].

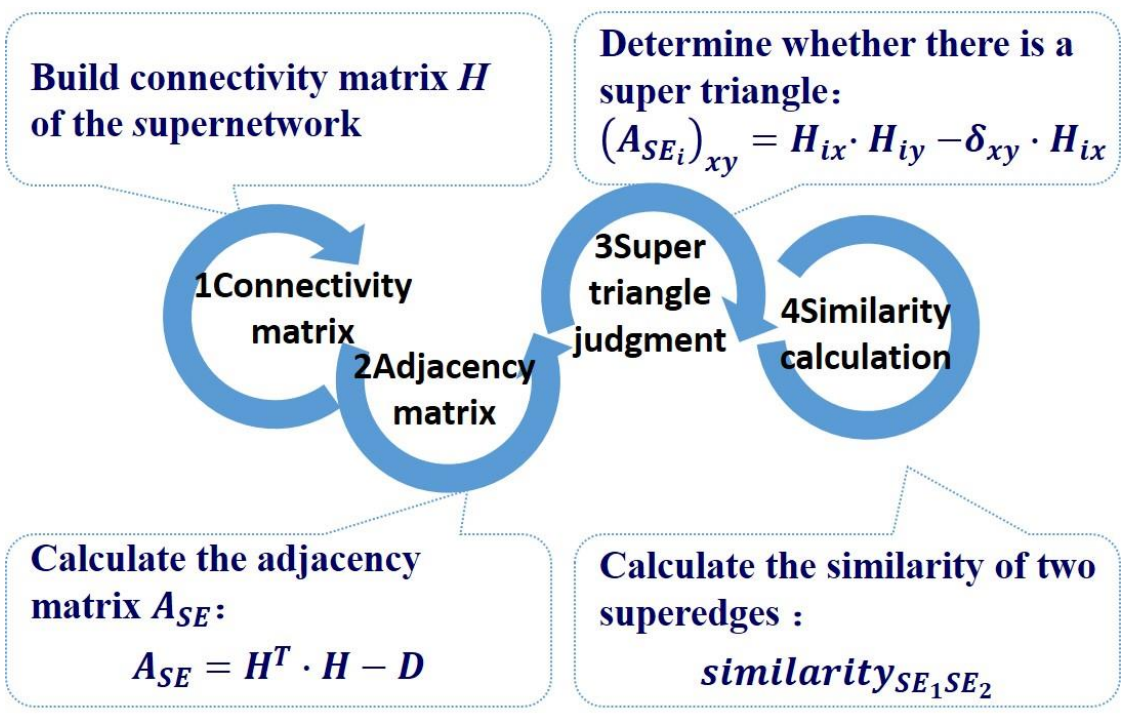

Fig. 3. Flow diagram of superlink prediction 
Through superlink prediction, we can know superedges that may appear in the next moment in opinion supernetwork. The predicted superedges contain opinion subjects, information environment, opinion psychology, and opinion viewpoints. This portrays how the subjects issued their point of view under an external driven force of environment and an internal driven force of psychology. And these viewpoints will in turn affect the development of events, becoming an output of the system. So we can predict the intervention timing of the system in accordance with opinion superlink prediction results. Suppose a supernetwork model of a public opinion system is as shown in Fig. 4. In the figure, red hollow graphics are those with negative attribute. Green solid graphics are those with positive attribute. The red dashed line represents a negative predicted superedge, and the green double-dashed line represents a positive predicted superedge. Intervention for the system is required when the predicted superedges in negative is more than positive ones.

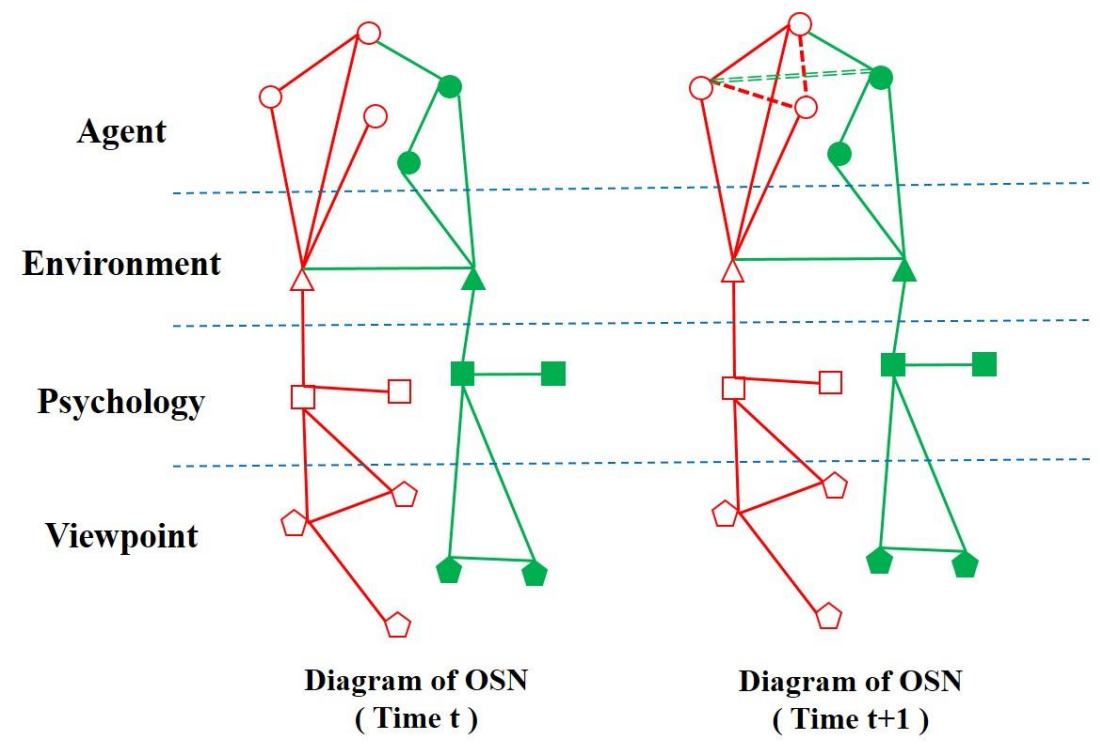

Fig. 4. Diagram of intervention timing

\subsection{Intervention Mode}

Experience has shown that when serious emergencies occur, the mainstream media should proactive and timely report the truth of emergencies objectively, with the support and guidance of the government and the propaganda department. This may 
inhibit the spread of rumors, and maintain social stability. Speaking of the mainstream media in the first time will resolve irrational pressure of public opinion. In the Internet era, it is fast and convenient for people accessing to information through the Internet, SMS and other channels. When a serious emergency occurs, the message will spread like wildfire, and it will have a strong psychological impact on the public in the short term. Mainstream media should occupy the initiative of the incident reporting.

Intervention of online public opinion system can be implemented by changing system input. Input of the system is the opinion environment, that is, different development stages of events. Thus changing opinion environment, i.e. publishing the latest information of the event, can promote the event to positive direction. This will achieve the intervention purpose of online public opinion system. After the release of the new positive information, the psychology of the opinion subjects will change under the impacts of the external driving force of opinion environment. Opinion subjects will update their opinion objects under the internal driving force of opinion psychology, namely the views expressed by opinion objects further varies with changes in their psychology. In Fig. 5, the bigger solid green triangle represents the newly added opinion environment. The green double-dashed line represents intervention of public opinion environment on the various elements of the system. It should be emphasized that environmental impact will be different between the opinion subjects in the center position of reply relationship in the system (core agent) and the ones in the edge position (leaf agent). So how to control the intervention intensity to get best effect under particular cost becomes the key point of the rumor intervention study. 


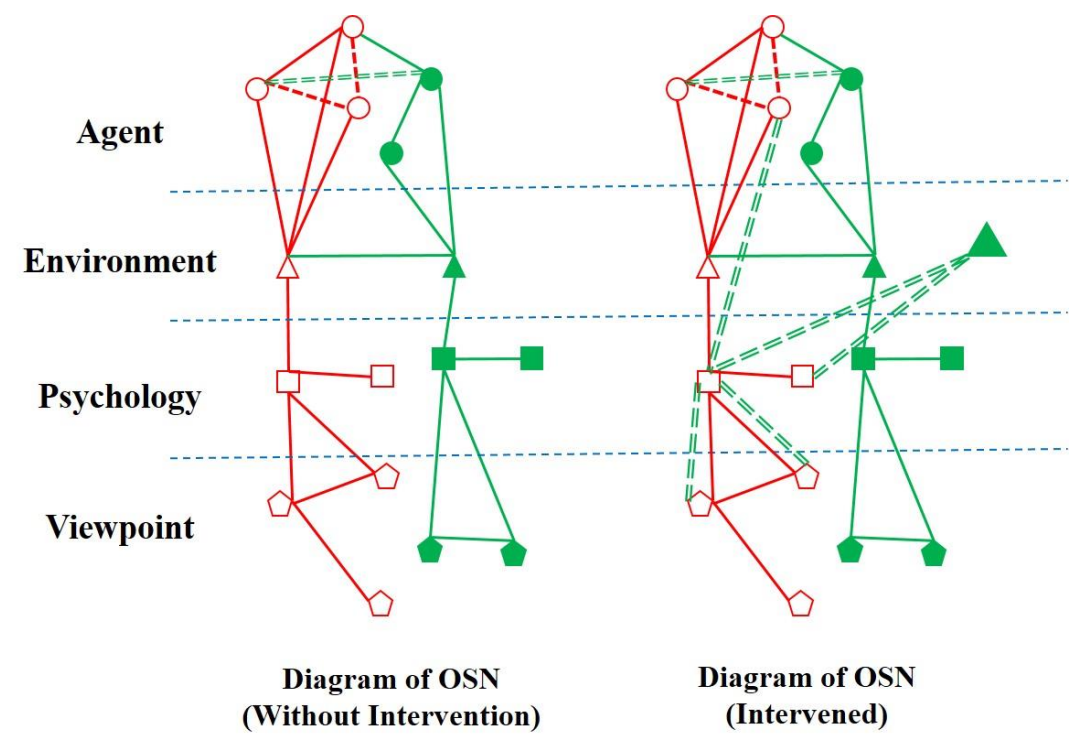

Fig. 5. Diagram of intervention mode

\subsection{Intervention Intensity}

Psychological state of public opinion subject is the dynamic of system evolution. Each subject will produce specific psychology in a particular environment, and published their viewpoints on social events under this psychological impact. Dynamic of system evolution is adjusted after subject's psychology changes, i.e. condition basis of viewpoints expressing is changed. Therefore, for public opinion system, intervention intensity must be strong enough to change the subject's psychology.

After the release of positive information to the public opinion system for intervention, the overall evolution rules of the model are: changes of the environment cause the changes of the subject's psychology; changes of the subject's psychology cause the changes of subject's attribute; changes of subject's attribute affect the attribute of newly published viewpoint. Specific changes of each Subnetwork in the model are as follows:

\subsubsection{Evolution of System Environment}

The new environment of agent $A_{i}$ is $E^{\prime}$. Its property depends on environment 
information $E$ and the neighbors of agent $A_{i}$ (i.e. other agents have replying relationships with $A_{i}$ ), namely,

$$
E^{\prime}=\frac{\partial_{E} \times N u m+\sum \partial_{j} \times 2}{N u m}
$$

Num is the neighbors' number of agent $A_{i} . \partial_{E}=+1$, it is the property of a new environmental information. $A_{j}$ are neighbors of agent $A_{i}, \partial_{j}$ are their points of view. If a viewpoint is a positive one, $\partial_{j}=+1$; otherwise $\partial_{j}=-1 \cdot j=1,2, \mathrm{~L}, N u m$, and $j \neq i$.

\subsubsection{Evolution of System Dynamics}

Opinion subject's psychology changes when the environment changes. If the subject is exposed to a changed environment, we may calculate the new environment of the agent in according to formula (2). And then we calculate the psychology transition probability under the new positive environment. The probability that the positive psychology of an agent's transform to a negative one is

$$
P(+\rightarrow-)=\frac{e^{\left(-E^{\prime} / C\right)}}{e^{\left(E^{\prime} / C\right)}+e^{\left(-E^{\prime} / C\right)}}
$$

And the probability that the negative psychology of an agent's transform to a positive one is

$$
P(-\rightarrow+)=\frac{e^{\left(E^{\prime} / C\right)}}{e^{\left(E^{\prime} / C\right)}+e^{\left(-E^{\prime} / C\right)}}
$$

$C$ reflects the concern degree on an event by the public.

\subsubsection{Evolution of Opinion Subjects}

The opinion subject property is judged according to the attribute of its psychology in accordance with the following rules: 
a. $\exists$ subject psychology is negative, the subject is negative.

b. $\forall$ subject psychology is positive, the subject is positive.

\subsubsection{Evolution of Opinion Objects}

Objects, i.e. viewpoints of the subject changes when the subject's property changed.

a. If the subject property changes from a negative one to a positive one, the negative standpoints of the subject change to the majority of the positive standpoints of the neighbors'.

b. If the subject property changes from a positive one to a negative one, the positive standpoints of the subject change to the majority of the negative standpoints of the neighbors'.

\section{Case Study}

\subsection{Experiment Design}

Matlab programming is used in this study to seek the variation of the proportion of positive agents over time under the release of positive information. We picked an agricultural rumor event as a study case. The model of the case contains four Subnetworks, including 108 nodes of agents in $G_{A}, 3$ nodes of environmental information in $G_{E}, 5$ nodes of psychological statuses in $G_{P}$, and 28 nodes of viewpoints in $G_{K}$.The relationships of nodes in and between different Subnetworks form the superedges of the Opinion SuperNetwork. This simulation experiment aims at analyzing how the proportion of positive agents change with time under the opinion intervention implement, and exploring the efficiency of rumor intervention based on the meta-synthetic methodology. Here we proposed three different intervention strategies, and compared the efficiency of various strategies. The experiment procedure is as follows:

Strategy 1. The released positive information affects all subjects at the same time.

Step 1: Initialize the property values of all nodes and the superedges of the Opinion SuperNetwork. 
Step 2: Calculate the positive agents' proportion. When the ratio is $<50 \%$, continue to next step, otherwise end.

Step 3: Simulate the release of a positive information. Add a positive environment node to simulate intervention implement.

Step 4: Choose a negative agent node randomly to update the node's property and other nodes' in the opinion supernetworks according to the evolution rules described in Section 3.4.

Step 5: Return to Step 2 and cycle.

Strategy 2. The released positive information affects core subjects at the first time. In Step 4, choose core subjects of negative property first.

Strategy 3. The released positive information affects leaf subjects at the first time.

In Step 4, choose leaf subjects of negative property first.

\subsection{Result Analysis}

Implement all the above three intervention strategies. Conduct 1000 simulation experiments independently under each of the strategies, and take the average value of the 1000 simulation experiments as the result (Fig.6).

As can be seen from Fig. 6, after the release of positive information, the proportion of positive public opinion gradually increased under all the three intervention strategies. It indicates that establishing supernetwork model based on the meta-synthetic methodology to conduct rumor interventions can achieve the desired outcomes. 


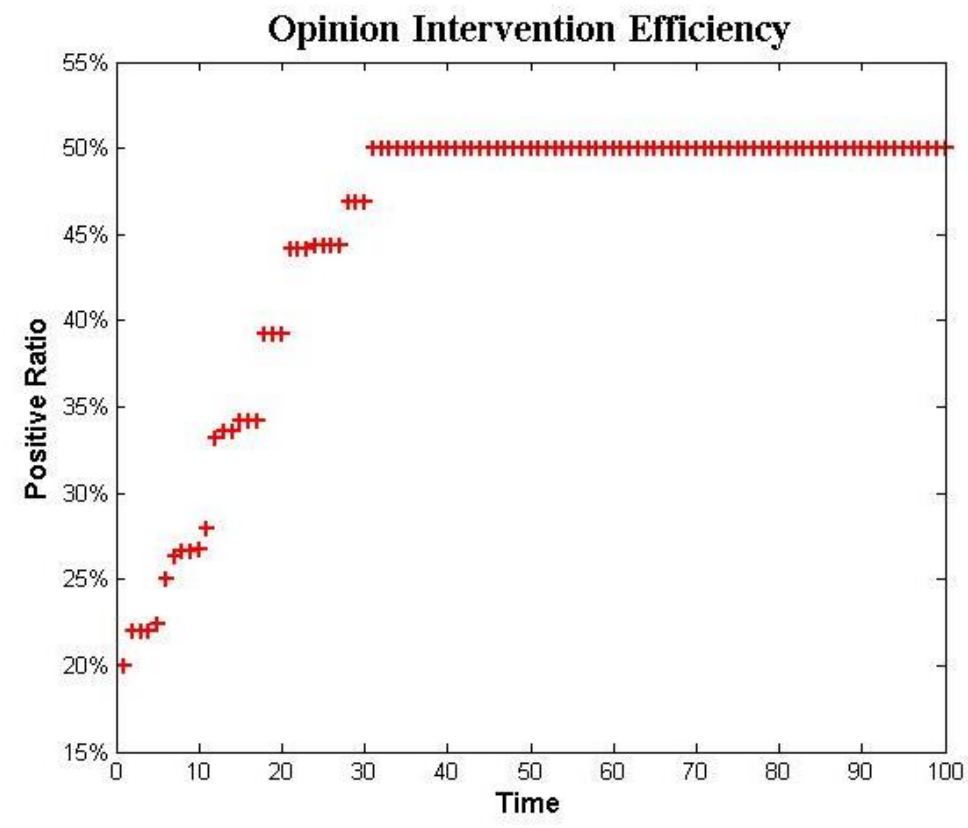

(a) Strategy 1. Both core and leaf agents at the same time

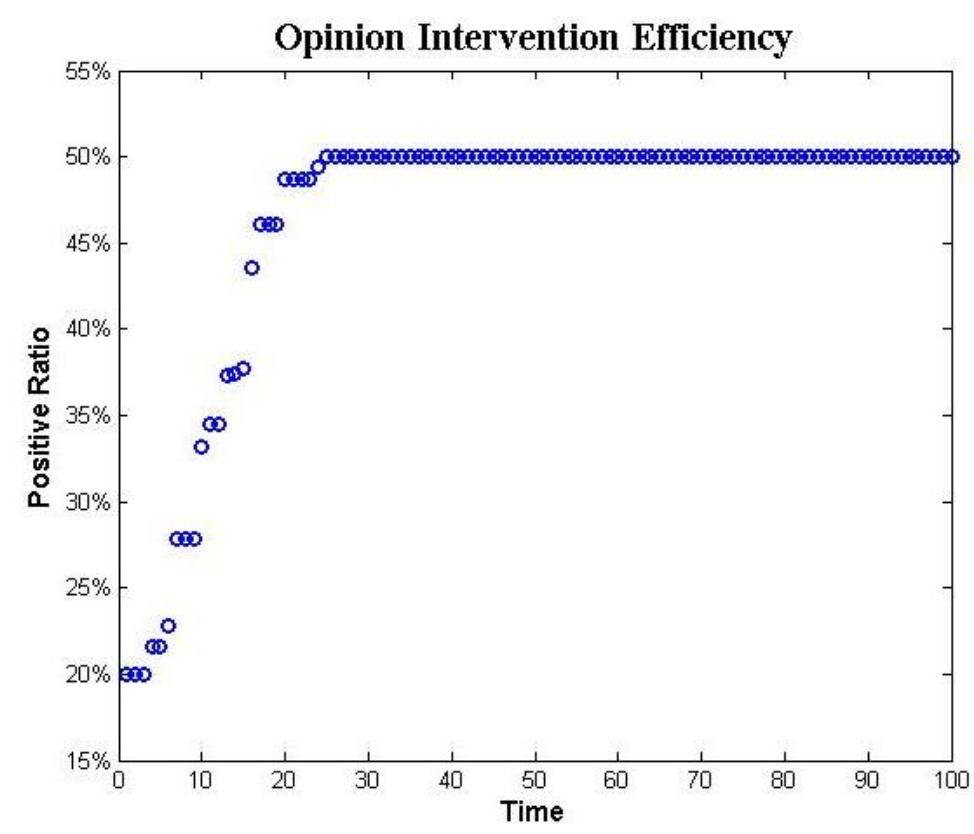

(b) Strategy 2. Core agents first 


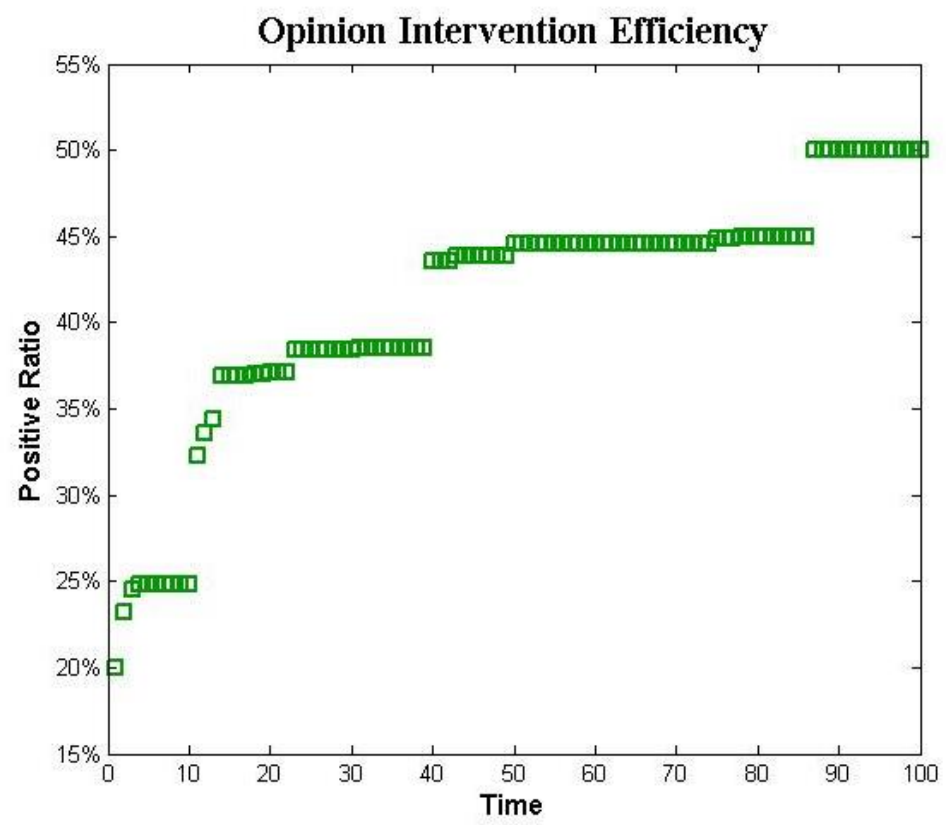

(c) Strategy 3. Leaf agents first

Fig. 6. Rumor Intervention Efficiency of Information Release

In addition, efficiency of the three different intervention strategies is in comparison (Fig. 7). In the beginning, the strategy that positive information first affects the of leaf public opinion agents has the highest efficiency, followed by the strategy that both kinds of opinion agents being affected at the same time. The strategy that information impacts core public opinion agents first is the least efficient. That may because the leaf agent kind is superior in numbers to core ones. Soon, the advantage of affecting core agents first shows up. By the 15th time step, effect of affecting core agents first surpasses the other two strategies. After the system is steady, strategy of affecting core agents first has a dominant effect, followed by the strategy that both kinds of opinion agents being affected at the same time.

It is commonly known that publishing positive information, and making it affect the core public opinion agents first is the best strategy for rumor intervention. This intervention mechanism research based on a meta-synthetic methodology approach has provided a reliable quantitative basis for evidence. However, it can be seen from the efficiency comparison of the three kinds of strategies that the efficiency of affecting all the public opinion agents together is only slightly inferior to the one of 
affecting core public opinion agents first. While in practice the letter takes a much higher cost. So in the real agricultural rumor intervention, take the strategy of affecting all public opinion agents at the same time can achieve a higher effect under a lower cost.

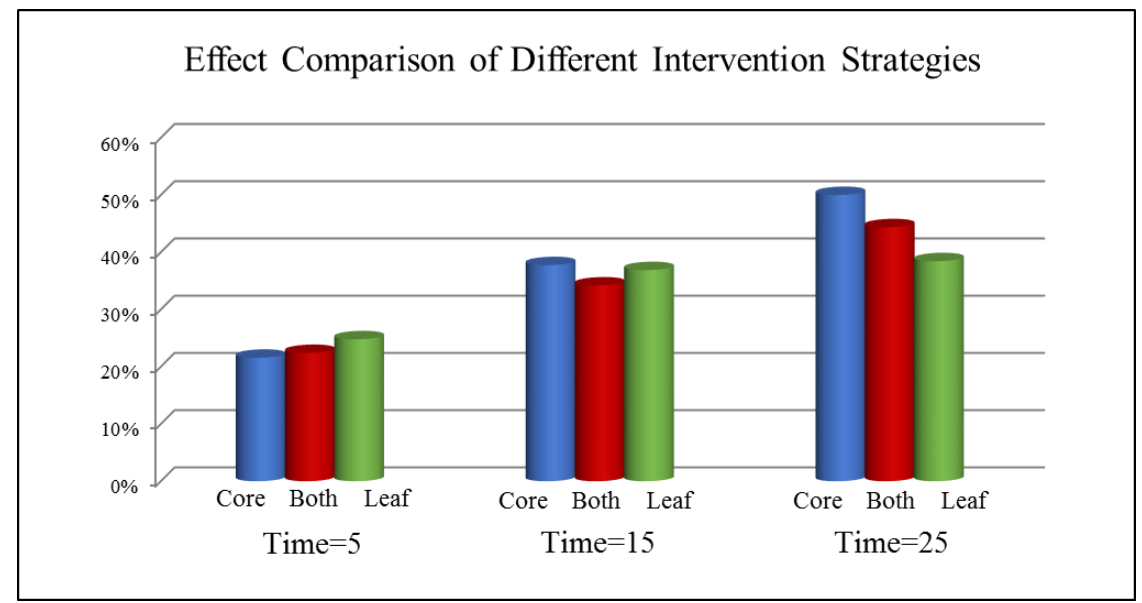

Fig. 7. Effect Comparison of Different Intervention Strategies

Simulation results show that analyzing rumor intervention mechanism from the perspective of meta-synthetic methodology to develop intervention strategies can not only quantify intervention strategies, but also draw influence mechanisms and laws of intervention strategies by changes and interlocking mechanisms of each element in the online public opinion system. This will help to provide a reliable basis for the development of more sophisticated and effective intervention strategies.

\section{Conclusions}

Meta-synthetic methodology provides a new perspective to study online agricultural rumor intervention. Online public opinion is seen as an organic integrity with specific functions composed of several elements of interaction and interdependence. This helps us to make a comprehensive analysis of the relationships among elements, elements and the system, as well as the system and its environment. Thus we can grasp its internal relationship and law, and achieve the purpose of controlling and transforming the system effectively.

Our investigation made two important contributions to the investigation of online agricultural rumor intervention. First, based on the meta-synthetic methodology, intervention mechanism of online public opinion system was investigated under a way 
of "from qualitative to quantitative". We described the general characteristics of online public opinion system qualitatively, including its composition, structure, and function. Then we built a supernetwork model which includes Social Subnetwork, Environment Subnetwork, Psychological Subnetwork and Viewpoint Subnetwork, to quantitatively research public opinion intervention mechanism to get the general intervention methods of public opinion system.

Second, this paper contributes to the research of online agricultural rumor intervention mechanism. We used superlink prediction to provide a theoretical basis for predicting the intervention timing. The release of new environmental information to impact the system input is used as the specific intervention mode. Changing the system dynamic by intervening in the psychological condition of the opinion agents was taken as the measure of intervention intensity. And the simulation results show that build a supernetwork model based on meta-synthetic methodology to implement rumor intervention can achieve desired effects. The analysis of the intervention strategies in this paper provides the government a comprehensive measure standard of online agricultural rumor events, which allows them to get a much deeper understanding to the rumor spreading influence elements, and make more strategic decisions intervening in rumor spreading.

Our study provides a useful consultation to agricultural rumor intervention theoretical studies from a meta-synthetic methodology perspective. And in future studies, more work may be done to improve our research. This article only analyzed the system changes caused by the environmental Subnetwork. The next step, we will continue to investigate the system changes caused by the other three Subnetworks, and also explore the corresponding intervention strategies.

\section{Acknowledgements}

Funds for this research was provided by the National Social Science Foundation of China (15CTQ030), the National Natural Science Foundation of China (91324009), the National Sci-Tech Pillar Program during the 12th Five-year Plan Period (2011BAH10B06), and the Science and Technology Innovation Projects of the Chinese Academy of Agricultural Sciences (CAAS). 


\section{References}

1. Y. J. Liu, Q. Q. Li, R. Y. Tian, N. Ma, Formation and application of public opinion based on supernetwork analysis, Bulletin of Chinese Academy of Sciences 28 (5) (2012) 560-568.

2. Q. Q. Li, Y. J. Liu, Dynamical model of public opinion and its application based on supernetwork, Bulletin of Chinese Academy of Sciences 28 (5) (2012) 569-577.

3. R. Y. Tian, Y. J. Liu, Intervention of public opinion and its application based on supernetwork analysis, Bulletin of Chinese Academy of Sciences 28 (5) (2012) 578-585.

4. N. Ma, Y. J. Liu, Recognition of online opinion leaders based on supernetwork analysis, Bulletin of Chinese Academy of Sciences 28 (5) (2012) 586-594.

5. R. Y. Tian, Z. L. Chen, Advances in intervention of online public opinion, Social Physics Series No. 4, Science Press, Beijing, 2013.

6. L. Von Bertalanffy, General system theory: Foundations, development, applications, New York, George Braziller, 1968.

7. X. S. Qian, A new discipline of science: the study of open complex giant system and its methodology, Urban Studies (5) (2005) 001.

8. J. F. Gu, Practice on Wuli-Shili-Renli system approach, Chinese Journal of Management 8 (3) (2011) 317-322, 355.

9. E. Katz, P. F. Lazarsfeld, Personal Influence, The part played by people in the flow of mass communications, New Brunswick, Transaction Publishers, 1970.

10. R. Y. Tian, Y. J. Liu, W. Y. Niu, Leader-Guiding model of online opinion supernetwork, Chinese Journal of Management Science 22 (10) (2014) 136-141.

11. Nagurney, On the relationship between supply chain and transportation network equilibria: a supernetwork equivalence with computations, Transportation Research Part E 42 (4) (2006) 293-316.

12. Y. J. Xi, Y. Z. Dang, K. J. Liao, Knowledge supernetwork model and its application in organizational knowledge systems, Journal of Management Sciences in China 12 (3) (2009) 12-21.

13. E. Estrada, J. A. Rodríguez-Velázquez, Subgraph centrality and clustering in complex hyper-networks, Physica A: Statistical Mechanics and its Applications 364 (2006) 581-594.

14. T. Wakolbinger, A. Nagurney, Dynamic supernetworks for the integration of social networks and supply chains with electronic commerce: modeling and analysis of buyer-seller relationships with computations, Netnomics 6 (2) (2004) 153-185.

15. Nagurney, J. Dong, Management of knowledge intensive systems as supernetworks: 
modeling, analysis, computations, and applications, Mathematical and Computer Modelling 42 (3-4) (2005) 397-417.

16. R. Y. Tian, Y. J. Liu, Isolation, insertion, and reconstruction: three strategies to intervene in rumor spread based on supernetwork model, Decision Support Systems 67 (2014) $121-130$.

17. R. Y. Tian, X. F. Zhang, Y. J. Liu, SSIC Model: a multi-layer model for intervention of online rumors spreading, Physica A: Statistical Mechanics and its Applications 427 (2015) 181-191.

18. Y. J. Liu, N. Ma, H. B. Wang, Study on the guidance of online public opinion for innovation social management, Bulletin of Chinese Academy of Sciences 27 (1) (2012) 9-16.

19. Y. J. Liu, Q. Q. Li, X. Y. Tang, N. Ma, R. Y. Tian, Superedge prediction, Management Review 24 (12) (2012) 137-145.

20. Y. J. Liu, Q. Q. Li, X. Y. Tang, N. Ma, R. Y. Tian, Superedge prediction: what opinions will be mined based on an opinion supernetwork model? Decision Support Systems 64 (2014) 118-129. 